\title{
Invariant Solutions and Bifurcation Analysis of the Nonlinear Transmission Line Model
}

\section{Sachin Kumar ( $\nabla$ sachin1jan@yahoo.com )}

Central University of Punjab https://orcid.org/0000-0001-6883-7788

\section{Research Article}

Keywords: Nonlinear Transmission lines, Power law nonlinearity, Lie symmetry method, Exact Solutions, Bifurcation analysis

Posted Date: March 16th, 2021

DOI: https://doi.org/10.21203/rs.3.rs-297502/v1

License: (c) (i) This work is licensed under a Creative Commons Attribution 4.0 International License. Read Full License 


\title{
Invariant solutions and bifurcation analysis of the nonlinear transmission line model
}

\author{
SACHIN KUMAR* \\ Department of Mathematics and Statistics \\ Central University of Punjab, Bathinda 151001, Punjab, India. \\ sachin1jan@yahoo.com
}

\begin{abstract}
In this paper, the nonlinear transmission line model with the power law nonlinearity and the constant capacitance and voltage relationship is studied using Lie symmetry analysis. Corresponding to the infinitesimals obtained, using commutation relations, abelian and non abelian Lie subalgebras are obtained. Also, using the adjoint table, the one dimensional optimal system of subalgebra is presented. Based on the optimal system, corresponding Lie symmetry reductions are obtained. Moreover, variety of new similarity solutions in the form of trigonometric functions, hyperbolic functions are obtained. Corresponding to one similarity reduction, by bifurcation of dynamical system, the stable and unstable regions are determined, which show the existence of soliton solutions from the nonlinear dynamics view point. Some of the obtained solutions are represented graphically and observations are also discussed.

Keywords: Nonlinear Transmission lines, Power law nonlinearity, Lie symmetry method, Exact Solutions, Bifurcation analysis.
\end{abstract}

\section{Introduction}

In physics, nonlinearity is observed in many areas of physics $2,10,11,13,18,21,25,28,31,32$. To understand the nonlinear phenomena, which are described by nonlinear parial differential equations (NLPDEs), we need to obtain their exact solutions. Recently, work has been done to study the exact solutions of these NLPDEs. Some of them include the Lie symmetry method [22,26], Direct method for symmetries [8], Non-classical symmetry method [3], Backlund transformation method [9], solitary wave ansatz method [4, Hirota's bilinear method [6, 15, 30], the modified simple equation method 17, the $\left(\frac{G^{\prime}}{G}\right)$ expansion method 33, and so on.

Among the methods in literature, the Lie symmetry method 16, 22, 26, 27, 29] is one of the most effective methods for finding the exact solutions of NLPDEs by which it is possible to reduce the number of independent variables and further to reduce the order of ordinary differential equations, thus making them easier to solve.

In electronic and communications engineering, a transmission line is a cable designed to conduct the currents with a frequency high enough that their wave nature must be taken into account. In this paper, We consider a non-linear transmission line 12 of the following form:

$$
L \frac{\partial}{\partial t}\left[C(v) \frac{\partial v}{\partial t}\right]=\frac{\partial^{2} v}{\partial x^{2}}+\frac{\delta^{2}}{12} \frac{\partial^{4} v}{\partial x^{4}}
$$

where $C$ and $L$ denote the capacitance(depends on voltage $v$ ) and inductance respectively. Afshari et al. 1] proposed and obtained travelling wave and numerical solutions of one and two dimensional non-uniform NLTL equations. El-Borai et al. 12 used extended tanh equation method to obtain

*sachin1jan@yahoo.com 
the soliton solutions of 11.1 for the relation $C(V)=C_{0}(1-b v)$. Mostafa 21] obtained analytical solutions of NLTL equation using improved tanh and sech methods for $C-V$ relationships $C(V)=$ $C_{0}(1-b v)$ and $C(V)=C_{0}\left(1+b_{1} v+b_{2} v^{2}\right)$.

In this work Lie point symmetry group classification [7, 23] of a class of nonlinear transmission line model with respect to capacitance and voltage relationship is discussed. Let us approximate the capacitor's voltage dependence in the form of power law nonlinearity, linear and constant relations as following:

$$
\begin{aligned}
(i) & C(v)=C_{0}(1-b v)^{n} \\
(\text { ii }) & C(v)=C_{0}
\end{aligned}
$$

which reduces the equation 1.1 to

$$
\begin{aligned}
(1-b v)^{n} \frac{\partial^{2} v}{\partial t^{2}}-n b(1-b v)^{n-1}\left(\frac{\partial v}{\partial t}\right)^{2} & =\frac{1}{L C_{0}} \frac{\partial^{2} v}{\partial x^{2}}+\frac{\delta^{2}}{12 L C_{0}} \frac{\partial^{4} v}{\partial x^{4}} \\
\frac{\partial^{2} v}{\partial t^{2}} & =\frac{1}{L C_{0}} \frac{\partial^{2} v}{\partial x^{2}}+\frac{\delta^{2}}{12 L C_{0}} \frac{\partial^{4} v}{\partial x^{4}}
\end{aligned}
$$

As mentioned above, some special cases of NLTL model without power law nonlinearity, were already discussed by several authors.

The main purpose of this paper is to obtain symmetry reductions and exact solutions to the nonlinear transmission lines for the capacitor's voltage dependence in the forms $(1.2)-(1.3)$. Firstly, symmetries of non-linear transmission lines (1.4)-(1.5), are obtained using the Lie classical method. Then, corresponding to the optimal systems, the symmetry reductions and exact solutions of the equations are obtained. Some graphical representations of obtained solutions are also given. Furthermore, for one reduction, the exact power series solution is investigated, and the convergence of the obtained power series solution is shown. Phase plane analysis of one of reduction is also performed.

\section{$2 \quad$ Lie symmetry analysis}

Let us first consider the Lie group of point transformations

$$
\begin{gathered}
x^{*}=x+\epsilon \xi(x, t, v)+O\left(\epsilon^{2}\right) \\
t^{*}=t+\epsilon \tau(x, t, v)+O\left(\epsilon^{2}\right) \\
v^{*}=v+\epsilon \eta(x, t, v)+O\left(\epsilon^{2}\right) .
\end{gathered}
$$

Let

$$
X=\xi_{i}(x, t, v) \frac{\partial}{\partial x_{i}}+\eta(x, t, v) \frac{\partial}{\partial v}
$$

be the infinitesimal generator of the Lie group of point transformations 2.6. Let

$$
\begin{aligned}
X^{(k)}= & \xi_{i}(x, t, v) \frac{\partial}{\partial x_{i}}+\eta(x, t, v) \frac{\partial}{\partial v}+\eta_{i}{ }^{(1)}(x, t, v, \partial v) \frac{\partial}{\partial v_{i}}+\ldots \\
& +\eta_{i_{1} i_{2} \ldots i_{k}}{ }^{(k)}\left(x, t, v, \partial v, \partial^{2} v, \ldots, \partial^{k} v\right) \frac{\partial}{\partial v_{i_{1} i_{2} \ldots i_{k}}}
\end{aligned}
$$

be the $k$-th extended infinitesimal generator of 2.7$)$. Then the one-parameter Lie group of point transformations 2.6 is admitted by $\operatorname{PDE} F\left(x, t, v, \partial v, \partial^{2} v, \ldots, \partial^{k} v\right)=0$, if and only if $X^{(k)} F\left(x, t, v, \partial v, \partial^{2} v, \ldots, \partial^{k} v\right)=0$ when $F\left(x, t, v, \partial v, \partial^{2} v, \ldots, \partial^{k} v\right)=0$. 


\subsection{Determination of Lie symmetries and optimal system for equation (1.4)}

Assuming the equation (1.4) is invariant under the transformations (2.6), the following invariance condition is obtain:

$$
\begin{aligned}
& (1-b v)^{n} \eta^{t t}-b n(1-b v)^{n-1} v_{t t} \eta-n(n-1) b^{2}(1-b v)^{n-2} v_{t}^{2} \eta-2 n b(1-b v)^{n-1} v_{t} \eta^{t} \\
& =\frac{1}{L C_{0}} \eta^{x x}+\frac{\delta^{2}}{12 L C_{0}} \eta^{x x x x}
\end{aligned}
$$

where $\eta^{t t}, \eta^{x x}, \eta^{x x x x}$ are prolonged infinitesimals acting on an extended space that contains all the derivatives of the dependent variable i.e., $v_{t t}, v_{x x}$, and $v_{x x x x}$.

The infinitesimal generator of a point symmetry admitted by equation 1.4 is of the form:

$$
X=\xi(x, t, v) \frac{\partial}{\partial x}+\tau(x, t, v) \frac{\partial}{\partial t}+\eta(x, t, v) \frac{\partial}{\partial v} .
$$

The values upto the fourth prolongation [5] are given by,

$$
\begin{array}{r}
\eta^{t t}=D_{t}\left(\eta^{t}\right)-v_{t x} D_{t} \xi-v_{t t}\left(D_{t} \tau\right), \\
\eta^{x x}=D_{x}\left(\eta^{x}\right)-v_{x x} D_{x} \xi-v_{x t}\left(D_{x} \tau\right), \\
\eta^{x x x x}=D_{x}\left(\eta^{x x x}\right)-v_{x x x x} D_{x} \xi-v_{x x x t}\left(D_{x} \tau\right) .
\end{array}
$$

Therefore, computing and substituting the values of $\eta^{t t}, \eta^{x x}$ and $\eta^{x x x x}$ in equation (2.9), we get the symmetry determining equations. Solving the determining equations, following expressions for the infinitesimals $\xi, \tau, \eta$ is obtained:

$$
\begin{aligned}
\xi(x, t, v) & =c_{2}, \\
\tau(x, t, v) & =c_{1}+t c_{3}, \\
\eta(x, t, v) & =\frac{2}{b n}(b v-1) c_{3},
\end{aligned}
$$

where $c_{1}, c_{2}, c_{3}$ are arbitrary parameters.

The point symmetry generators admitted by equation (1.4) are given by

$$
\begin{aligned}
& X_{1}=\frac{\partial}{\partial t} \\
& X_{2}=\frac{\partial}{\partial x} \\
& X_{3}=t \frac{\partial}{\partial t}+\frac{2}{b n}(b v-1) \frac{\partial}{\partial v} .
\end{aligned}
$$

The commutator table of the generators is given by Table 1 .

\begin{tabular}{|c|c|c|c|}
\hline & $X_{1}$ & $X_{2}$ & $X_{3}$ \\
\hline$X_{1}$ & 0 & 0 & $X_{1}$ \\
\hline$X_{2}$ & 0 & 0 & 0 \\
\hline$X_{3}$ & $-X_{1}$ & 0 & 0 \\
\hline
\end{tabular}

Table 1: Commutator Table

From the table, it can be seen that the generators form a Lie algebra. 


\begin{tabular}{|c|c|c|c|}
\hline & $X_{1}$ & $X_{2}$ & $X_{3}$ \\
\hline$X_{1}$ & $X_{1}$ & $X_{2}$ & $X_{3}-\epsilon X_{1}$ \\
\hline$X_{2}$ & $X_{1}$ & $X_{2}$ & $X_{3}$ \\
\hline$X_{3}$ & $X_{1} e^{\epsilon}$ & $X_{2}$ & $X_{3}$ \\
\hline
\end{tabular}

Table 2: Adjoint Table

From commutator table, one can see that $X_{1}, X_{2}$ generate abelian Lie subalgebra and $X_{1}, X_{3}$ forms non-abelian Lie subalgebra 24. The adjoint representation 22 is given by:

$\operatorname{Ad}\left(\exp \left(\epsilon X_{i}\right) X_{j}\right)=X_{j}-\epsilon\left[X_{i}, X_{i}\right]+\frac{\epsilon^{2}}{2 !}\left[X_{i},\left[X_{i}, X_{j}\right]\right]-\ldots$

The Lie algebra $\mathcal{L}^{3}$ is solvable and the adjoint table of the generators is given as in Table 2 .

Theorem 1.: An optimal system [22] of Lie algebras is provided by:

(i) $X_{3}+\lambda X_{2}$, (ii) $X_{2}+\mu X_{1}$, (iii) $X_{1}$.

Proof: Consider the symmetry algebra of the Eq. 1.4 whose adjoint representation is determined in Table 2 ,

Let us consider the non-zero vector in the form

$$
X=a_{1} X_{1}+a_{2} X_{2}+a_{3} X_{3} .
$$

We need to simplify this vector field by using suitable adjoint maps. First assume that $a_{3} \neq 0$ and assume $a_{3}=1$.

Now if we apply on $X$ by $\operatorname{Ad}\left(\exp \left(a_{1} X_{3}\right)\right) X$, using the table 2 the coefficient of $X_{1}$ will vanish as follows:

$$
A d\left(e^{a_{1} X_{3}}\right) X=a_{2} X_{2}+X_{3} .
$$

It cannot be reduced further by applying adjoint operations. So, $X$ is equivalent to $a_{2} X_{2}+X_{3}$ under the adjoint representation. The remaining subalgebras can be spanned by vectors of the form $X$ with $a_{3}=0$. If $a_{2} \neq 0$, we may take $a_{2}=1$, and then we have

$$
X^{\prime}=a_{1} X_{1}+X_{2}
$$

We can further act on $X^{\prime}$ by the group generated by $X_{3}$, this has the effect of scaling the coefficient of $X_{1}$ :

$$
\operatorname{Ad}\left(e^{\epsilon X_{3}}\right) X^{\prime}=a_{1} e^{\epsilon} X_{1}+X_{2} .
$$

Thus any sub-algebra spanned by $X$ with $a_{3}=0, a_{2} \neq 0$ is equivalent to one spanned by either $a_{1} e^{\epsilon} X_{1}+X_{2}$, where $a_{1}$ is arbitrary and may take values $+1,-1$ or 0 . Further assuming $a_{3}=a_{2}=0$ and $a_{1}=1$ we left with subalgebra $X_{1}$.

\subsection{Determination of Lie symmetries for equation 1.5}

As procedure mentioned above, for equation 1.5 , we get rich class of symmetries and the infinitesimals $\xi, \tau$ and $\eta$ are obtained as follows:

$$
\begin{aligned}
\xi(x, t, u)= & C_{2} \\
\tau(x, t, u)= & C_{1} \\
\eta(x, t, u)= & \left(C_{6} t+C_{11}\right) \cos \left(\frac{2 \sqrt{3}}{\delta} x\right)+\left(C_{5} t+C_{10}\right) \sin \left(\frac{2 \sqrt{3}}{\delta} x\right) \\
& +\left(C_{4} x+C_{3}\right) t+C_{9} x+C_{7} v+C_{8},
\end{aligned}
$$


where $C_{1}, C_{2}$ and $C_{3}$ are arbitrary constants.

The eleven dimensional symmetry algebra admitted by equation 1.5 is given by

$$
\begin{aligned}
X_{1} & =\frac{\partial}{\partial t}, X_{2}=\frac{\partial}{\partial x}, X_{3}=t \frac{\partial}{\partial v}, X_{4}=x t \frac{\partial}{\partial v} \\
X_{5} & =t \sin \left(\frac{2 \sqrt{3}}{\delta} x\right) \frac{\partial}{\partial v}, X_{6}=t \cos \left(\frac{2 \sqrt{3}}{\delta} x\right) \frac{\partial}{\partial v} \\
X_{7} & =v \frac{\partial}{\partial v}, X_{8}=\frac{\partial}{\partial v}, X_{9}=x \frac{\partial}{\partial v} \\
X_{10} & =\sin \left(\frac{2 \sqrt{3}}{\delta} x\right) \frac{\partial}{\partial v}, X_{11}=\cos \left(\frac{2 \sqrt{3}}{\delta} x\right) \frac{\partial}{\partial v} .
\end{aligned}
$$

In this case, the non zero commutator relations are obtained as follows:

$$
\begin{aligned}
& {\left[X_{1}, X_{3}\right]=X_{8},\left[X_{1}, X_{4}\right]=X_{9},\left[X_{1}, X_{5}\right]=X_{10},\left[X_{1}, X_{6}\right]=X_{11}} \\
& {\left[X_{2}, X_{4}\right]=X_{3},\left[X_{2}, X_{5}\right]=\frac{2 \sqrt{3}}{\delta} X_{6},\left[X_{2}, X_{6}\right]=-\frac{2 \sqrt{3}}{\delta} X_{5}} \\
& {\left[X_{2}, X_{9}\right]=X_{8},\left[X_{2}, X_{10}\right]=\frac{2 \sqrt{3}}{\delta} X_{11},\left[X_{2}, X_{11}\right]=-\frac{2 \sqrt{3}}{\delta} X_{10},} \\
& {\left[X_{3}, X_{7}\right]=X_{3},\left[X_{4}, X_{7}\right]=X_{4},\left[X_{5}, X_{7}\right]=X_{5},\left[X_{6}, X_{7}\right]=X_{6}} \\
& {\left[X_{7}, X_{8}\right]=-X_{8},\left[X_{7}, X_{9}\right]=-X_{9},\left[X_{7}, X_{10}\right]=-X_{10},\left[X_{7}, X_{11}\right]=-X_{11}}
\end{aligned}
$$

So Lie algebra generated by vector fields $X_{i}, i=1,2, \ldots, 11$, is non-abelian.

For reduction, we will consider the following linear combinations of vector fields:

$$
\begin{aligned}
& \text { (i) } X_{1}+\mu X_{2}+\lambda X_{7}, \\
& \text { (ii) } X_{1}+\mu X_{2}+C_{3} X_{3}+C_{9} X_{9} \\
& \text { (iii) } X_{1}+\mu X_{2}+C_{5} X_{5}+C_{6} X_{6}+C_{6} X_{10}+C_{5} X_{11} \text {, }
\end{aligned}
$$

where $\mu, \lambda, C_{3}, C_{5}, C_{6}, C_{9}$ are arbitrary constants.

\section{Symmetry Reductions}

In this section, the symmetry reductions of non-linear transmission line equations $(1.4)-(1.5)$ are obtained.

\subsection{Similarity reductions for equation 1.4}

As explained in section-2.1 optimal system of vector fields for equation 1.4 is:

(i) $X_{3}+\lambda X_{2}$ (ii) $X_{2}+\mu X_{1}$, (iii) $X_{1}$.

For vector field $X_{3}+\lambda X_{2}$

Corresponding to this vector field, solving the characteristic equation, we have following similarity variables

$$
\begin{aligned}
s & =t e^{-\frac{x}{\lambda}} \\
v(x, t) & =F(s) e^{\frac{2 x}{n \lambda}}+\frac{1}{b},
\end{aligned}
$$


where $s$ is new independent variable and $F$ is new dependent variable.

Using (3.22) in equation (1.4), we obtain following ODE

$$
\begin{aligned}
& \left((-1)^{n} L C_{0} b^{n} \lambda^{4} F^{n}+s^{2}\left(\frac{2}{n} \delta^{2}-\frac{7}{12} \delta^{2}-\lambda^{2}-\frac{2}{n^{2}} \delta^{2}\right)\right) F^{\prime \prime}+(-1)^{n} n L C_{0} \lambda^{4} b^{n} F^{n-1} F^{2} \\
& +\left(\frac{8 \delta^{2}}{3 n^{3}}-\frac{2 \delta^{2}}{n^{2}}+\frac{4 \lambda^{2}}{n}+\frac{2 \delta^{2}}{3 n}-\lambda^{2}-\frac{\delta^{2}}{12}\right) s F^{\prime}-\frac{\delta^{2}}{12} s^{4} F^{\prime \prime \prime \prime}+\delta^{2}\left(\frac{2}{3 n}-\frac{1}{2}\right) s^{3} F^{\prime \prime \prime} \\
& -\frac{4}{n^{2}}\left(\lambda^{2}+\frac{\delta^{2}}{3 n^{2}}\right) F=0
\end{aligned}
$$

where' denotes derivatives with respect to $s$.

For vector field $X_{2}+\mu X_{1}$

For vector field $X_{2}+\mu X_{1}$, similarity variables are

$$
\begin{aligned}
\xi & =x-\mu t \\
v(x, t) & =G(\xi),
\end{aligned}
$$

where $\xi$ and $G$ are corresponding new variables.

Using (3.24) in (1.4), we have

$$
\left((1-b G)^{n} G^{\prime}\right)^{\prime}-\frac{1}{\mu^{2} L C_{0}} G^{\prime \prime}-\frac{\delta^{2}}{12 \mu^{2} L C_{0}} G^{\prime \prime \prime \prime}=0,
$$

where' denotes derivate with respect to $\xi$.

\section{For vector field $X_{1}$}

Corresponding to this vector field, similarity variables are as follows:

$$
\begin{aligned}
\tau & =x \\
v & =H(\tau),
\end{aligned}
$$

where $\tau$ is new independent variable and $H$ is new dependent variable.

Using (3.26), in (1.4), we have

$$
H^{\prime \prime}+\frac{\delta^{2}}{12} H^{\prime \prime \prime \prime}=0,
$$

where ' denotes derivatives with respect to $\tau$.

\subsection{Similarity reduction for equation 1.5}

For vector field $X_{1}+\mu X_{2}+\lambda X_{7}$

Solving the characteristic equations for the vector field 2.18$)$, we have following similarity variables

$$
\begin{aligned}
\xi & =x-\mu t \\
v(x, t) & =F(\xi) e^{\lambda t},
\end{aligned}
$$

where $\xi$ and $F$ are new independent variable and dependent variables, respectively.

Using (3.28) in 1.5), we have following ODE

$$
\begin{aligned}
& \left(-\delta^{2} \lambda^{4}-12 \lambda^{2}\right) F(\xi)+\left(-4 \delta^{2} \lambda^{3}-24 \lambda\right) \frac{\mathrm{d}}{\mathrm{d} \xi} F(\xi)+\left(12 C_{1} L \mu^{2}-6 \delta^{2} \lambda^{2}-12\right) \frac{\mathrm{d}^{2}}{\mathrm{~d} \xi^{2}} F(\xi) \\
& -4\left(\frac{\mathrm{d}^{3}}{\mathrm{~d} \xi^{3}} F(\xi)\right) \delta^{2} \lambda-\left(\frac{\mathrm{d}^{4}}{\mathrm{~d} \xi^{4}} F(\xi)\right) \delta^{2}=0 .
\end{aligned}
$$


For vector field $X_{1}+\mu X_{2}+C_{3} X_{3}+C_{9} X_{9}$

For vector field (2.19), we have following similarity variable of the equation 1.5

$$
\begin{aligned}
\xi & =x-\mu t \\
v(x, t) & =\frac{C_{9} x^{2}}{2 \mu}+\frac{C_{3}}{2} t^{2}+G(\xi) .
\end{aligned}
$$

Using 3.30 in 1.5 , we have

$$
L C O\left(C_{3}+\left(\frac{\mathrm{d}^{2}}{\mathrm{~d} \xi^{2}} G(\xi)\right) \mu^{2}\right)-\frac{C_{9}}{\mu}-\frac{\mathrm{d}^{2}}{\mathrm{~d} \xi^{2}} G(\xi)-\frac{\delta^{2}}{12}\left(\frac{\mathrm{d}^{4}}{\mathrm{~d} \xi^{4}} G(\xi)\right)=0 .
$$

For vector field $X_{1}+\mu X_{2}+C_{5} X_{5}+C_{6} X_{6}+C_{6} X_{10}+C_{5} X_{11}$

For vector field 2.20 , we have following similarity variable of the equation 1.5 .

$$
\begin{aligned}
\xi & =x-\mu t \\
v(x, t) & =\frac{\sqrt{3} \delta t}{6}\left(-C_{5} \cos \left(2 \frac{\sqrt{3} x}{\delta}\right)+C_{6} \sin \left(2 \frac{\sqrt{3} x}{\delta}\right)\right)+G(\xi) .
\end{aligned}
$$

Using (3.32) into the main equation 1.5 , we have following ODE

$$
L C 0\left(\frac{\mathrm{d}^{2}}{\mathrm{~d} \xi^{2}} G(\xi)\right) \mu^{2}-\frac{\delta^{2}}{12}\left(\frac{\mathrm{d}^{4}}{\mathrm{~d} \xi^{4}} G(\xi)\right)-\frac{\mathrm{d}^{2}}{\mathrm{~d} \xi^{2}} G(\xi)=0 .
$$

\section{Exact solutions}

In this section we will obtain the exact solutions of the equations 1.4 -1.5.

\subsection{For equation 1.4}

For finding the solutions of 1.4 , firstly we will solve the equation (3.23).

Let us assume the solution of equation $(3.23)$ in the following form

$$
F(\sigma)=k s^{p},
$$

where $k$ and $p$ have to be determined.

Using (4.34) in 3.23 and simplifying, we get the following values

$$
\begin{aligned}
p & =1 /(1+n) \\
\lambda & = \pm \frac{\iota(n+1) \delta}{2 \sqrt{3} n(n+1)} .
\end{aligned}
$$

Using (4.34) and 4.35) in 3.22, solution of equation (3.23) is given by

$$
v(x, t)=k t^{\frac{1}{1+n}}\left[\cos \left(\frac{2 \sqrt{3}}{\delta} x\right) \pm \iota \sin \left(\frac{2 \sqrt{3}}{\delta} x\right)\right]+\frac{1}{b} .
$$

For solving the equation (3.25), substitute

$$
1-b G(\sigma)=V(\sigma)
$$

we obtain the following ODE

$$
\frac{\delta^{2}}{12} V^{\prime \prime \prime \prime}-\mu^{2} C_{0} L\left(V^{n} V^{\prime}\right)^{\prime}+V^{\prime \prime}=0 .
$$


Now integrating 4.38 twice and taking constant of integration equal to zero, we have

$$
\frac{\delta^{2}}{12} V^{\prime \prime}-\mu^{2} C_{0} L \frac{V^{n+1}}{n+1}+V=0
$$

Balancing the highest derivative terma and nonlinear term, we have $m+2=(n+1) m$. So that

$$
m=\frac{2}{n} .
$$

Clearly $m$ is integer only if $n=1$ or $n=2$. Case $n=1$, will be discussed separately. For all other values of $n$, Let us apply the transformation

$$
V(\sigma)=W(\sigma)^{\frac{1}{n}}
$$

Using 4.41, equation 4.39, reduces to

$$
n \delta^{2}(n+1) W W^{\prime \prime}-12 \mu^{2} C 0 L n^{2} W^{3}+12 n^{2}(n+1) W^{2}-\delta^{2}\left(n^{2}-1\right) W^{\prime 2}=0 .
$$

Now for obtaining solutions corresponding to $\mathrm{ODE}(\sqrt{4.42})$ ), we will apply extended $\left(\frac{G^{\prime}}{G}\right)$ - expansion method $[14$.

\subsubsection{Method Description}

Let us consider a general nonlinear PDE with constant coefficients:

$$
F\left(u, u_{x}, u_{t}, u_{x x}, u_{t t}, u_{x t}, \ldots\right)=0
$$

where $u=u(x, t)$ is an unknown function, $F$ is polynomial in $u(x, t)$ and its partial derivatives, in which the highest derivative term and nonlinear terms are involved. Following are main steps involved.

Step 1. To reduce the equation 4.43 into ordinary differential equation (ODE), I consider a variable $\xi$ such that

$$
u(x, t)=u(\xi), \quad \xi=x-\mu t,
$$

where $\mu$ is speed of travelling wave.

Using 4.44 into 4.43), I have

$$
F\left(u, u^{\prime}, u^{\prime \prime}, \ldots\right)=0,
$$

where' denotes derivative with respect to $\xi$.

Step 3. Let us consider the solution of equation 4.45 in following form:

$$
\begin{array}{r}
u(\xi)=a_{0}+\sum_{i=0}^{m}\left[a_{i}\left(\frac{G^{\prime}}{G}\right)^{i}+b_{i}\left(\frac{G^{\prime}}{G}\right)^{i-1} \sqrt{\rho\left(1+\frac{1}{\mu_{1}}\left(\frac{G^{\prime}}{G}\right)^{2}\right)}\right. \\
\left.+c_{i}\left(\frac{G^{\prime}}{G}\right)^{-i}+d_{i} \frac{\left(\frac{G^{\prime}}{G}\right)^{-i+1}}{\sqrt{\rho\left(1+\frac{1}{\mu_{1}}\left(\frac{G^{\prime}}{G}\right)^{2}\right)}}\right],
\end{array}
$$

where $a_{i}, b_{i}, c_{i}, d_{i}(i=1, \ldots, m)$ are arbitrary constants to be determined later, $\rho= \pm 1, m$ is positive integer, and $G=G(\xi)$ satisfies the following ODE

$$
G^{\prime \prime}+\mu_{1} G=0
$$

where $\mu_{1}$ is to be determined.

Step 4. Now determine the value of $m$ by homogeneous balance between the highest derivative 
term and highest nonlinear term for equation 4.45.

Step 5. Substitute (4.46) with 4.47) into equation 4.45), we have a equation in powers of

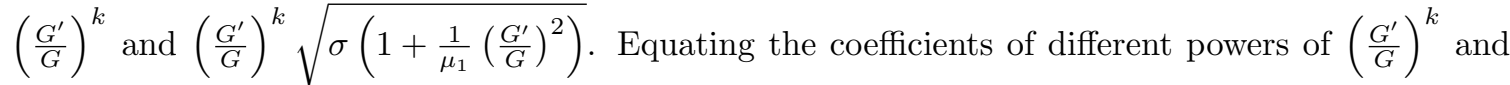
$\left(\frac{G^{\prime}}{G}\right)^{k} \sqrt{\sigma\left(1+\frac{1}{\mu_{1}}\left(\frac{G^{\prime}}{G}\right)^{2}\right)}$ equal to zero, yeild system of algebraic equations. Solving this system of equation, we obtain values of parameters $\mu, \mu_{1}, a_{i}, b_{i}, c_{i}, d_{i}(i=1, \ldots, m)$.

Step 6. Substituting the value of different parameters $\mu, \mu_{1}, a_{i}, b_{i}, c_{i}, d_{i}(i=1, \ldots, m)$ with solutions of equation (4.47) into (4.46), we obtain different travelling wave solutions of equation 4.43).

Using the general solution of equation 4.47) in 4.46), we have the following values of $\frac{G}{G}$ :

Family 1 . When $\mu_{1}<0$ then we have hyperbolic type solutions as follows:

$$
\frac{G^{\prime}}{G}=\sqrt{-\mu_{1}} \frac{C_{1} \sinh \left(\sqrt{-\mu_{1}} \xi\right)+C_{2} \cosh \left(\sqrt{-\mu_{1}} \xi\right)}{C_{1} \cosh \left(\sqrt{-\mu_{1}} \xi\right)+C_{2} \sinh \left(\sqrt{-\mu_{1}} \xi\right)}
$$

where $C_{1}$ and $C_{2}$ are two arbitrary constants.

Family 2. When $\mu_{1}>0$ then we have trigonometric type solutions as follows:

$$
\frac{G^{\prime}}{G}=\sqrt{\mu_{1}} \frac{C_{1} \cos \left(\sqrt{\mu_{1}} \xi\right)-C_{2} \sin \left(\sqrt{-\mu_{1}} \xi\right)}{C_{1} \sin \left(\sqrt{\mu_{1}} \xi\right)+C_{2} \cos \left(\sqrt{\mu_{1}} \xi\right)}
$$

where $C_{1}$ and $C_{2}$ are two arbitrary constants.

Family 3. If $\mu_{1}=0$ then we have rational type solutions as follows:

$$
H(\xi)=\frac{C_{1}}{C_{2}+C_{1} \xi}
$$

where $C_{1}$ and $C_{2}$ are two arbitrary constants.

Now we will apply the method to ODE (4.42). Balancing the highest order derivative term and nonlinear term in the equation 4.42 , we have $m=2$.

So, as per algorithm for the extended $\left(\frac{G^{\prime}}{G}\right)$ expansion method, value of solution $W(\sigma)$ of equation 4.42 is given by

$$
\begin{array}{r}
W(\sigma)=a_{0}+\sum_{i=0}^{2}\left[a_{i}\left(\frac{G^{\prime}}{G}\right)^{i}+b_{i}\left(\frac{G^{\prime}}{G}\right)^{i-1} \sqrt{\rho\left(1+\frac{1}{\mu_{1}}\left(\frac{G^{\prime}}{G}\right)^{2}\right)}\right. \\
\left.+c_{i}\left(\frac{G^{\prime}}{G}\right)^{-i}+d_{i} \frac{\left(\frac{G^{\prime}}{G}\right)^{-i+1}}{\sqrt{\rho\left(1+\frac{1}{\mu_{1}}\left(\frac{G^{\prime}}{G}\right)^{2}\right)}}\right]
\end{array}
$$

where $G$ satisfy the Eq. (4.47) with $\xi$ replaced by $\sigma, \rho= \pm 1, a_{0}, a_{1}, a_{2}, b_{1}, b_{2}, c_{1}, c_{2}, d_{1}, d_{2}$ are to be determined.

Substituting (4.51) in 4.42 with 4.47) and taking coefficients of $\left(\frac{G^{\prime}}{G}\right)^{k}$ and $\left(\frac{G^{\prime}}{G}\right)^{k} \sqrt{\rho\left(1+\frac{1}{\mu_{1}}\left(\frac{G^{\prime}}{G}\right)^{2}\right)}$ equal to zero, yield a set of algebraic equations in $a_{0}, a_{1}, a_{2}, b_{1}, b_{2}, c_{1}, c_{2}, d_{1}, d_{2}, \mu_{1}$ and $\mu$. Solving the system of algebraic equation using the software Maple, we have following results:

\section{Set 1:}

$\mu=\frac{3 n^{2}}{\delta^{2} \mu_{1}}, a_{0}=\frac{\left(n^{2}+3 n+2\right) \delta^{4} \mu_{1}^{2}}{18 n^{4} L C 0}, c_{2}=\frac{\delta^{4} \mu_{1}^{3}\left(n^{2}+3 n+2\right)}{18 n^{4} L C 0}, a_{1}=a_{2}=b_{1}=b_{2}=c_{1}=c_{2}=d_{1}=d_{2}=$ 0 , and $\mu_{1}$ is arbitrary, 


\section{Set 2:}

$\mu=\frac{3 n^{2}}{\delta^{2} \mu_{1}}, a_{0}=\frac{\left(n^{2}+3 n+2\right) \delta^{4} \mu_{1}^{2}}{18 n^{4} L C 0}, a_{2}=\frac{\left(n^{2}+3 n+2\right) \delta^{4} \mu_{1}}{18 n^{4} L C 0}, a_{1}=b_{1}=b_{2}=c_{1}=c_{2}=d_{1}=d_{2}=0$, and $\mu_{1}$ is arbitrary,

\section{Set 3:}

$\mu=\frac{3 n^{2}}{4 \delta^{2} \mu_{1}}, a_{0}=\frac{4\left(n^{2}+3 n+2\right) \delta^{4} \mu_{1}{ }^{2}}{9 n^{4} L C 0}, a_{2}=\frac{2\left(n^{2}+3 n+2\right) \delta^{4} \mu_{1}}{9 n^{4} L C 0}, c_{2}=\frac{2 \delta^{4} \mu_{1}{ }^{3}\left(n^{2}+3 n+2\right)}{9 n^{4} L C 0}, a_{1}=b_{1}=b_{2}=c_{1}=$ $d_{1}=d_{2}=0$ and $\mu_{1}$ is arbitrary,

\section{Set 4:}

$\mu=\frac{12 n^{2}}{\delta^{2} \mu_{1}}, a_{0}=\frac{\left(n^{2}+3 n+2\right) \delta^{4} \mu_{1}^{2}}{144 n^{4} L C 0}, a_{2}=\frac{(n+1) \mu_{1} \delta^{4}(n+2)}{144 n^{4} L C 0}, b_{2}= \pm \frac{(n+1) \mu_{1} \delta^{4}(n+2)}{144 n^{4} L C 0} \sqrt{\frac{\mu}{\sigma}}, a_{1}=b_{1}=c_{1}=c_{2}=$ $d_{1}=d_{2}=0$ and $\mu_{1}$ is arbitrary.

One can see that $\mu_{1}$ is arbitrary in all Sets of values of $a_{0}, a_{1}, a_{2}, b_{1}, b_{2}, c_{1}, c_{2}, d_{1}, d_{2}$ and $\mu$. So, on depending upon the values of $\mu_{1}$, three cases of solutions can be derived as follows:

\section{(I) Hyperbolic solutions (if $\mu_{1}<0$ )}

If $\mu_{1}<0$ in sets $1,2,3$ and 4 , then using the Eq. (4.47) and transformation (4.41), we obtain following solutions of ODE 4.39 :

Set 1 and Set 2 give:

$$
V(\sigma)=\left\{\frac{(n+1)(n+2) \mu_{1}^{2} \delta^{4}}{18 n^{4} L C 0}\left(1-\left(\frac{-C 1 \sinh \left(\sqrt{-\mu_{1}} \sigma\right)+{ }_{-} C 2 \cosh \left(\sqrt{-\mu_{1}} \sigma\right)}{{ }_{-} C 1 \cosh \left(\sqrt{-\mu_{1}} \sigma\right)+{ }_{-} C 2 \sinh \left(\sqrt{-\mu_{1}} \sigma\right)}\right)^{2}\right)\right\}^{\frac{1}{n}} .
$$

Set 3 gives:

$$
\begin{aligned}
V(\sigma)=\left\{\frac{2 \delta^{4}(n+2)(n+1) \mu_{1}^{2}}{9 C 0 L n^{4}}\right. & \left(2-\left(\frac{{ }_{-} C 1 \cosh \left(\sqrt{-\mu_{1}} \sigma\right)+{ }_{-} C 2 \sinh \left(\sqrt{-\mu_{1}} \sigma\right)}{{ }_{-} C 1 \sinh \left(\sqrt{-\mu_{1}} \sigma\right)+{ }_{-} C 2 \cosh \left(\sqrt{-\mu_{1}} \sigma\right)}\right)^{2}\right. \\
& \left.\left.-\left(\frac{{ }_{-C 1} \cosh \left(\sqrt{-\mu_{1}} \sigma\right)+{ }_{-} C 2 \sinh \left(\sqrt{-\mu_{1}} \sigma\right)}{-C 1 \sinh \left(\sqrt{-\mu_{1}} \sigma\right)+{ }_{-} C 2 \cosh \left(\sqrt{-\mu_{1}} \sigma\right)}\right)^{-2}\right)\right\}^{\frac{1}{n}} .
\end{aligned}
$$

Set 4 gives

$$
\begin{aligned}
& V(\sigma)=\left\{a _ { 0 } \left(1-\left(\frac{{ }_{-} C 1 \sinh \left(\sqrt{-\mu_{1}} \sigma\right)+{ }_{-} C 2 \cosh \left(\sqrt{-\mu_{1}} \sigma\right)}{{ }_{-} C 1 \cosh \left(\sqrt{-\mu_{1}} \sigma\right)+{ }_{-} C 2 \sinh \left(\sqrt{-\mu_{1}} \sigma\right)}\right)^{2}\right.\right. \\
& \pm\left(\frac{-C 1 \sinh \left(\sqrt{-\mu_{1}} \sigma\right)+{ }_{-} C 2 \cosh \left(\sqrt{-\mu_{1}} \sigma\right)}{{ }_{-} C 1 \cosh \left(\sqrt{-\mu_{1}} \sigma\right)+{ }_{-} C 2 \sinh \left(\sqrt{-\mu_{1}} \sigma\right)}\right) \\
& \left.\left.\sqrt{-1+\left(\frac{-C 1 \sinh \left(\sqrt{-\mu_{1}} \sigma\right)+{ }_{-} C 2 \cosh \left(\sqrt{-\mu_{1}} \sigma\right)}{{ }_{-} C 1 \cosh \left(\sqrt{-\mu_{1}} \sigma\right)+{ }_{-} C 2 \sinh \left(\sqrt{-\mu_{1}} \sigma\right)}\right)^{2}}\right)\right\}^{\frac{1}{n}},
\end{aligned}
$$

where $a_{0}$ is given by set $4, \mu_{1}$ is arbitrary negative constant and $\sigma=x-\left(\frac{12 n^{2}}{\delta^{2} \mu_{1}}\right) t$.

Using the transformations (3.24) and 4.37), with above obtained solutions, corresponding solutions of main Eq. 1.4, can be obtained.

(II) Trigonometric solutions (if $\mu_{1}>0$ )

If $\mu_{1}>0$ in sets $1,2,3$ and 4, then using the Eq. 4.47) and transformation 4.41, we obtain following solutions of ODE (4.39): 
Set 1 and Set 2 give:

$$
V(\sigma)=\left\{\frac{(n+1)(n+2) \mu_{1}^{2} \delta^{4}}{18 n^{4} L C 0}\left(1+\left(\frac{{ }_{-} C 1 \sin \left(\sqrt{\mu_{1}} \sigma\right)+{ }_{-} C 2 \cos \left(\sqrt{\mu_{1}} \sigma\right)}{{ }_{-} C 1 \cos \left(\sqrt{\mu_{1}} \sigma\right)-{ }_{-} C 2 \sin \left(\sqrt{\mu_{1}} \sigma\right)}\right)^{2}\right)\right\}^{\frac{1}{n}} .
$$

Set 3 gives:

$$
\begin{aligned}
V(\sigma)=\left\{\frac{2 \delta^{4}(n+2)(n+1) \mu_{1}^{2}}{9 C 0 L n^{4}}\right. & \left(2+\left(\frac{-C 1 \cos \left(\sqrt{\mu_{1}} \sigma\right)-{ }_{-} C 2 \sin \left(\sqrt{\mu_{1}} \sigma\right)}{-C 1 \sin \left(\sqrt{\mu_{1}} \sigma\right)+{ }_{-} C 2 \cos \left(\sqrt{\mu_{1}} \sigma\right)}\right)^{2}\right. \\
& \left.+\left(\frac{-C 1 \cos \left(\sqrt{\mu_{1}} \sigma\right)-{ }_{-} C 2 \sin \left(\sqrt{\mu_{1}} \sigma\right)}{-C 1 \sin \left(\sqrt{\mu_{1}} \sigma\right)+{ }_{-} C 2 \cos \left(\sqrt{\mu_{1}} \sigma\right)}\right)\right\}^{-2} .
\end{aligned}
$$

Set 4 gives

$$
\begin{aligned}
& V(\sigma)=\left\{a _ { 0 } \left(1+\left(\frac{{ }_{-} C 1 \sin \left(\sqrt{\mu_{1}} \sigma\right)+{ }_{-} C 2 \cos \left(\sqrt{\mu_{1}} \sigma\right)}{{ }_{-} C 1 \cos \left(\sqrt{\mu_{1}} \sigma\right)-{ }_{C} C 2 \sin \left(\sqrt{\mu_{1}} \sigma\right)}\right)^{2}\right.\right. \\
& \pm\left(\frac{{ }_{-} C 1 \sin \left(\sqrt{\mu_{1}} \sigma\right)+{ }_{-} C 2 \cos \left(\sqrt{\mu_{1}} \sigma\right)}{{ }_{-} C 1 \cos \left(\sqrt{\mu_{1}} \sigma\right)-{ }_{-} C 2 \sin \left(\sqrt{\mu_{1}} \sigma\right)}\right) \\
& \left.\sqrt{1+\left(\frac{-C 1 \sin \left(\sqrt{\mu_{1}} \sigma\right)+{ }_{-} C 2 \cos \left(\sqrt{\mu_{1}} \sigma\right)}{{ }_{-} C 1 \cos \left(\sqrt{\mu_{1}} \sigma\right)-{ }_{-} C 2 \sin \left(\sqrt{\mu_{1}} \sigma\right)}\right)}\right\}^{2},
\end{aligned}
$$

where $a_{0}$ is given by set $4, \mu_{1}$ is arbitrary negative constant and $\sigma=x-\left(\frac{12 n^{2}}{\delta^{2} \mu_{1}}\right) t$.

Using the transformations (3.24) and (4.37), with above obtained solutions, corresponding solutions of main Eq. 1.4, can be obtained.

(III) Rational solutions (if $\mu_{1}=0$ )

For rational solutions, put $\mu_{1}=0$ and also taking $\mu=0$, in sets $1,2,3$ and 4 , we obtain trivial solution of ODE 4.39).

Using the transformations (3.24) and 4.37), with above obtained trivial solution, corresponding constant solution of main Eq. (1.4), can be obtained as follows:

$$
v(x, t)=\frac{1}{b} .
$$

\section{Particular cases:}

For particular values of $\_C 1$ and $\_C 2$ in aformentioned solutions, soliton, periodic and complex solutions can be obtained when parameters take special values as follows:

\section{(i) Soliton solutions}

Taking ${ }_{-}$2 $=0$ in 4.52, we obtain the following solitary wave solution

$$
V(\sigma)=\left\{\frac{(n+1)(n+2) \mu_{1}^{2} \delta^{4}}{18 n^{4} L C 0} \operatorname{sech}^{2}\left(\sqrt{-\mu_{1}} \sigma\right)\right\}^{\frac{1}{n}} .
$$




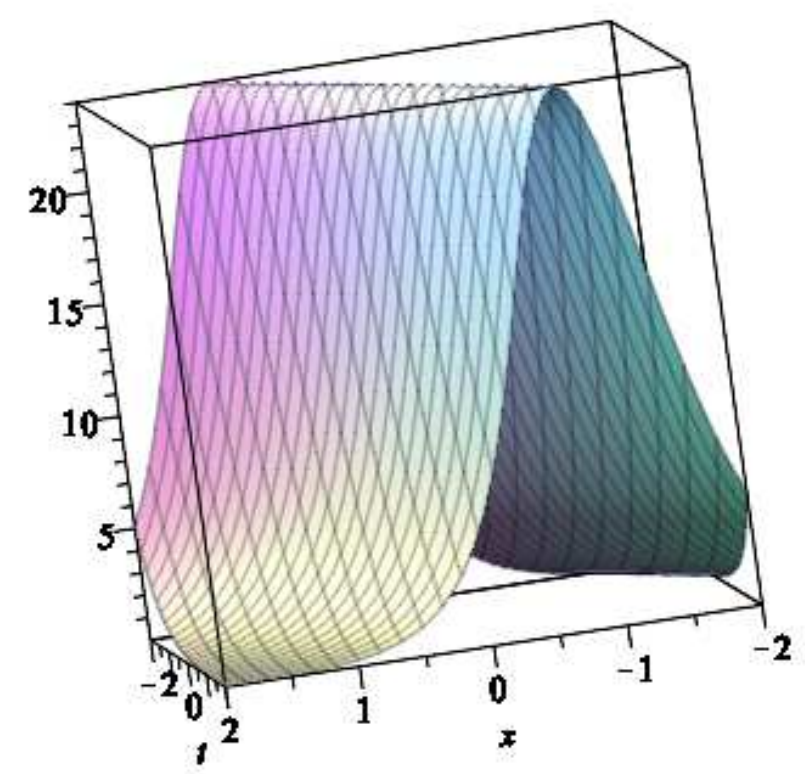

Figure 1: Soliton solution 4.59

Taking $\_1=0$ in 4.52 , following solitary wave solution is obtained

$$
V(\sigma)=\left\{-\frac{(n+1)(n+2) \mu_{1}^{2} \delta^{4}}{18 n^{4} L C 0} \operatorname{csch}^{2}\left(\sqrt{-\mu_{1}} \sigma\right)\right\}^{\frac{1}{n}} .
$$

Taking either ${ }_{-} C 1=0$ or ${ }_{-} C 2=0$ in 4.53 , we have following solitary wave solution

$$
V(\sigma)=\left\{\frac{(n+1)(n+2) \mu_{1}^{2} \delta^{4}}{18 n^{4} L C 0}\left(\operatorname{sech}^{2}\left(\sqrt{-\mu_{1}} \sigma\right)-\operatorname{csch}^{2}\left(\sqrt{-\mu_{1}} \sigma\right)\right)\right\}^{\frac{1}{n}} .
$$

Now taking _ $C 1=0$ in 4.54 , we have

$$
V(\sigma)=\left\{a_{0} \operatorname{csch}^{2}\left(\sqrt{-\mu_{1}} \sigma\right)\left(1 \pm \cosh \left(\sqrt{-\mu_{1}} \sigma\right)\right)\right\}^{\frac{1}{n}} .
$$

\section{(ii) Periodic solutions}

Setting $-C 1=0$ in 4.55 , we have

$$
V(\sigma)=\left\{\frac{(n+1)(n+2) \mu_{1}^{2} \delta^{4}}{18 n^{4} L C 0} \csc ^{2}\left(\sqrt{\mu_{1}} \sigma\right)\right\}^{\frac{1}{n}} .
$$

Setting ${ }_{-} 22=0$ in 4.55 , we have

$$
V(\sigma)=\left\{\frac{(n+1)(n+2) \mu_{1}^{2} \delta^{4}}{18 n^{4} L C 0} \sec ^{2}\left(\sqrt{\mu_{1}} \sigma\right)\right\}^{\frac{1}{n}} .
$$


Taking either ${ }_{-} C 1=0$ or ${ }_{-} C 2=0$ in 4.56 , we have following solitary wave solution

$$
V(\sigma)=\left\{\frac{(n+1)(n+2) \mu_{1}^{2} \delta^{4}}{18 n^{4} L C 0}\left(\sec ^{2}\left(\sqrt{\mu_{1}} \sigma\right)+\csc ^{2}\left(\sqrt{\mu_{1}} \sigma\right)\right)\right\}^{\frac{1}{n}} .
$$

Takinng _ $C 1=0$ in 4.57), we have

$$
V(\sigma)=\left\{a_{0} \csc ^{2}\left(\sqrt{\mu_{1}} \sigma\right)\left(1 \pm \cos \left(\sqrt{\mu_{1}} \sigma\right)\right)\right\}^{\frac{1}{n}} .
$$

Takinng_C2 $=0$ in (4.57), we have

$$
V(\sigma)=\left\{a_{0} \sec ^{2}\left(\sqrt{\mu_{1}} \sigma\right)\left(1 \pm \sin \left(\sqrt{\mu_{1}} \sigma\right)\right)\right\}^{\frac{1}{n}} .
$$

(iii) Complex solutions

Now taking _ $C 2=0$ in 4.54 , we have

$$
V(\sigma)=\left\{a_{0} \operatorname{sech}^{2}\left(\sqrt{-\mu_{1}} \sigma\right)\left(1 \pm \iota \sinh \left(\sqrt{-\mu_{1}} \sigma\right)\right)\right\}^{\frac{1}{n}} .
$$

Now solution of equation (3.27) is given by

$$
H(\tau)=C_{1}+C_{2} \tau+C_{3} \sin \left(2 \frac{\sqrt{3} \tau}{\delta}\right)+C_{4} \cos \left(2 \frac{\sqrt{3} \tau}{\delta}\right) .
$$

Corresponding solution of main equation $(1.4)$ is given by

$$
v(x, t)=C_{1}+C_{2} x+C_{3} \sin \left(2 \frac{\sqrt{3} x}{\delta}\right)+C_{4} \cos \left(2 \frac{\sqrt{3} x}{\delta}\right) .
$$

This is stationary solution of the equation.

\subsection{For equation 1.5}

Solution of ODE (3.29) can be obtained in the following form

$$
F(\xi)=\sum_{i=0}^{3} e^{r_{i} \xi}
$$

where $r_{i}, i=0,1,2,3$ are roots of the following polynomial in $m$

$$
\begin{array}{r}
-\delta^{2} \lambda^{4}-12 \lambda^{2}+\left(-4 \delta^{2} \lambda^{3}-24 \lambda\right) m+\left(12 C_{1} L \mu^{2}-6 \delta^{2} \lambda^{2}-12\right) m^{2} \\
-4 m^{3} \delta^{2} \lambda-m^{4} \delta^{2}=0 .
\end{array}
$$

Corresponding solution of equation 1.5 , can be given as

$$
v(x, t)=\sum_{i=0}^{3} e^{r_{i}(x-\mu t)+\lambda t},
$$

where $r_{i}, i=0,1,2,3$ are roots of the equation 4.72. 
Solution of 3.31 is obtained as

$$
\begin{aligned}
G(\xi) & =-1 / 4 \frac{-A 2 \delta^{2}}{-3 L C 0 \mu^{2}+3} \sin \left(2 \frac{\sqrt{-3 L C_{0} \mu^{2}+3} \xi}{\delta}\right) \\
& -1 / 4 \frac{-A 1 \delta^{2}}{-3 L C_{0} \mu^{2}+3} \cos \left(2 \frac{\sqrt{-3 L C_{0} \mu^{2}+3} \xi}{\delta}\right) \\
& +1 / 2 \frac{\left(-C_{0} L \mu C_{3}+C_{9}\right) \xi^{2}}{\mu\left(L C_{0} \mu^{2}-1\right)}+{ }_{-} A 3 \xi+{ }_{-} A 4,
\end{aligned}
$$

where _ $A 1, \_A 2, \_A 3$ and $\_A 4$ are arbitrary constants.

Using (3.30 and (4.74), solution of equation 1.5 is given as

$$
\begin{aligned}
v(x, t) & =\frac{C_{9} x^{2}}{2 \mu}+\frac{C_{3}}{2} t^{2}-\frac{-A 2 \delta^{2}}{-12 L C_{0} \mu^{2}+12} \sin \left(2 \frac{\sqrt{-3 L C_{0} \mu^{2}+3}(x-\mu t)}{\delta}\right) \\
& -\frac{-A 1 \delta^{2}}{-12 L C_{0} \mu^{2}+12} \cos \left(2 \frac{\sqrt{-3 L C_{0} \mu^{2}+3}(x-\mu t)}{\delta}\right) \\
& +\frac{\left(-C_{0} L \mu C_{3}+C_{9}\right)(x-\mu t)^{2}}{2 \mu\left(L C_{0} \mu^{2}-1\right)}+{ }_{-} A 3(x-\mu t)+{ }_{-} A 4 .
\end{aligned}
$$

Solution 4.74 is represented by the Fig. 2 .

\section{Observation}

One can observe that:

(i) As $\xi \rightarrow \pm \infty$, graph of solution is parabolic as in Fig. 2(a).

(ii) For transient value of $\xi$, solution is represented by bell shaped solitons as in Fig. 2(b).

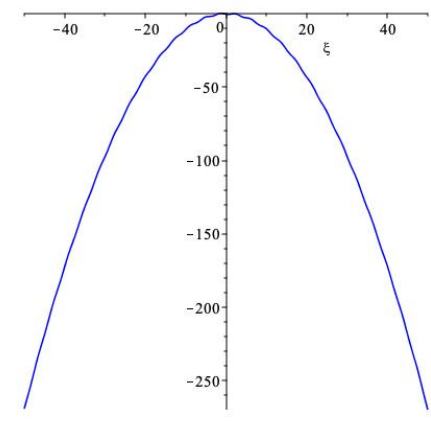

(a) As $\xi \rightarrow \pm \infty$

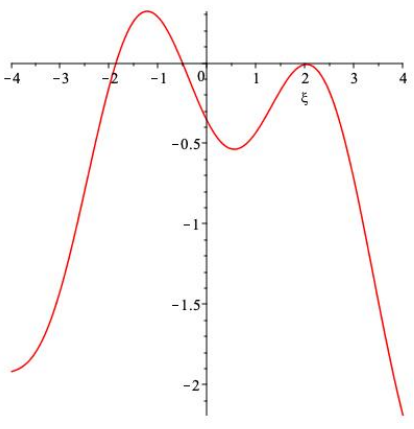

(b) For transient value of $\xi$

Figure 2: For $A_{1}=A_{2}=C_{9}=C_{3}=\delta=C_{0}=1, L=1.2, \mu=0.8$, graphs of the solutions 4.74).

Now let us obtain the solution of ODE 3.33. Using MAPLE, we obtain following solutions of this ODE

$$
G(\xi)=C_{1}+C_{2} \xi+C_{3} \sin \left(2 \frac{\sqrt{-3 L C_{0} \mu^{2}+3} \xi}{\delta}\right)+C_{4} \cos \left(2 \frac{\sqrt{-3 L C_{0} \mu^{2}+3} \xi}{\delta}\right) .
$$


Corresponding solution of 1.5$)$ is given by

$$
\begin{aligned}
v(x, t)= & \frac{\sqrt{3} \delta t}{6}\left(-C_{5} \cos \left(2 \frac{\sqrt{3} x}{\delta}\right)+C_{6} \sin \left(2 \frac{\sqrt{3} x}{\delta}\right)\right)+C_{1} \\
& +C_{2}(x-\mu t)+C_{3} \sin \left(2 \frac{\sqrt{-3 L C_{0} \mu^{2}+3}(x-\mu t)}{\delta}\right) \\
& +C_{4} \cos \left(2 \frac{\sqrt{-3 L C_{0} \mu^{2}+3}(x-\mu t)}{\delta}\right) .
\end{aligned}
$$

Solution 4.76 is represented by the Fig. 3.

\section{Observation}

One can observe that:

(i) With taking coefficient of $x i$ non-zero, the graph of the solution is parabolic as in Fig. 3 (a).

(ii) For taking coeffiecient of $\xi$ equal to zero, the graph of the solution is periodic as shown in Fig. 3 (b).

(iii) Also with increasing the value of $\mu$, the amplitude of travelling wave remains same but width increases as shown in Fig 3 (b).

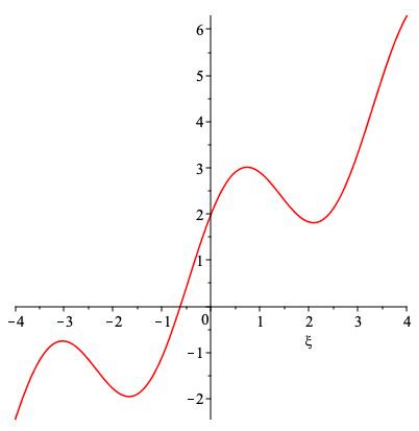

(a) Quasi periodic solution

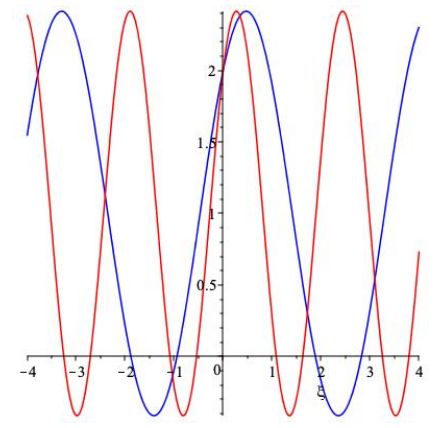

(b) Periodic solution

Figure 3: Graphs of the solution 4.76) (a) For $A_{1}=A_{2}=A_{3}=A_{4}=\delta=C_{0}=1, L=1.2, \mu=0.8$ (b) For $A_{1}=A_{3}=A_{4}=\delta=C_{0}=1, A_{2}=0, L=1.2$ and $\mu=0.5, \mu=0.8$ is shown corresponding to red, blue colors, respectively.

\section{Bifurcation Analysis}

In this section, the transmission line model with power law nonlinearity will be studied using the dynamical system approach. The phase portraits will be displayed.

\subsection{Bifurcation phase portraits and qualitative analysis}

Introducing the notation $X=V, Y=V^{\prime}$, let us reduce the Eq. 4.39 to the autonomous system:

$$
\begin{aligned}
X^{\prime} & =Y \\
Y^{\prime} & =\frac{a_{1}}{a_{0}} X^{n+1}-\frac{1}{a_{0}} X,
\end{aligned}
$$


where $a_{0}=\frac{\delta^{2}}{12}$ and $a_{1}=\frac{\mu^{2} C_{0} L}{n+1}$.

The system (5.78) is a Hamiltonian system with following Hamiltonian function:

$$
H(X, Y)=Y^{2}-\frac{2 a_{1}}{a_{0}(n+2)} X^{n+2}+\frac{1}{a_{0}} X^{2} .
$$

If the system is not Hamiltonian, then there is no closed orbit in the phase portrait. So in that case, system will not have solitary and periodic wave solutions. For obtaining the phase portrait of (5.78), set

$$
f(X)=\frac{a_{1}}{a_{0}} X^{n+1}-\frac{1}{a_{0}} X .
$$

When $n$ is even number and $a_{1}>0, f(X)$ has three zeros, $X_{-}, X_{0}$ and $X_{+}$given as follows:

$$
X_{-}=-\left(\frac{1}{a_{1}}\right)^{\frac{1}{n}}, X_{0}=0, X_{+}=\left(\frac{1}{a_{1}}\right)^{\frac{1}{n}} .
$$

When $n$ is odd number, $f(X)$ has two zeros, $X_{0}$ and $X_{*}$ given as follows:

$$
X_{0}=0, X_{*}=\left(\frac{1}{a_{1}}\right)^{\frac{1}{n}} .
$$

Let $\left(X_{i}, 0\right)$ be the critical point of 5.78 , then the corresponding characteristic values of linearized system (5.78) at the singular points $\left(X_{i}, 0\right)$ are given by

$$
\lambda_{ \pm}= \pm \sqrt{f^{\prime}\left(X_{i}\right)}
$$

Using the qualitative theory of dynamical system, we know that

1. If $f^{\prime}\left(X_{i}\right)>0,\left(X_{i}, 0\right)$ is saddle point.

2. If $f^{\prime}\left(X_{i}\right)<0,\left(X_{i}, 0\right)$ is a center point.

3. If $f^{\prime}\left(X_{i}\right)=0,\left(X_{i}, 0\right)$ is degenerate saddle point.

So, the bifurcation phase portraits of the system (5.78), are obtained as in Figs. 4 and 5 . If $n$ is even and $n a_{0}>0,(0,0)$ is center point and $\left(-\left(\frac{1}{a_{1}}\right)^{\frac{1}{n}}, 0\right),\left(\left(\frac{1}{a_{1}}\right)^{\frac{1}{n}}, 0\right)$ are unstable saddle point for system (5.78) as shown in Fig. 4a. For $n$ is odd and $n a_{0}>0,(0,0)$ is center point and $\left(\left(\frac{1}{a_{1}}\right)^{\frac{1}{n}}, 0\right)$ are unstable saddle point for system (5.78) as shown in Fig. $4 \mathrm{~b}$

For even $n$ and $n a_{0}<0,(0,0)$ is saddle point and $\left(-\left(\frac{1}{a_{1}}\right)^{\frac{1}{n}}, 0\right),\left(\left(\frac{1}{a_{1}}\right)^{\frac{1}{n}}, 0\right)$ are center points. For odd $n$ and $n a_{0}<0,(0,0)$ is saddle point and $\left(\left(\frac{1}{a_{1}}\right)^{\frac{1}{n}}, 0\right)$ is center point.

Let us consider

$$
H(X, Y)=h,
$$

where $h$ is Hamiltonian. Now, we will study the relation between the system (5.78) and the Hamiltonian $h$.

Set

$$
h^{*}=\left|H\left(X_{+}, 0\right)\right|=\left|H\left(X_{-}, 0\right)\right|=\left|H\left(X_{*}, 0\right)\right| .
$$

From Fig. 4 4 we have following results.

Proposition 5.1. When $n$ is even number and $n a_{0}>0$, we have

(I) When $h=0$, system (5.78) has two periodic orbits $P 1$ and $P 1^{*}$. 


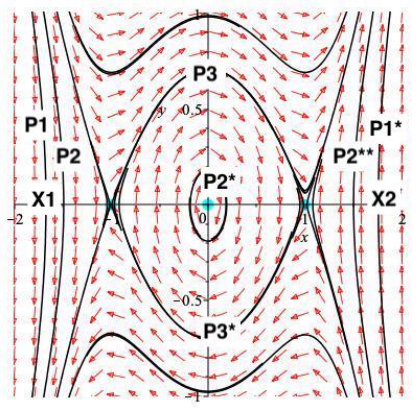

(a)

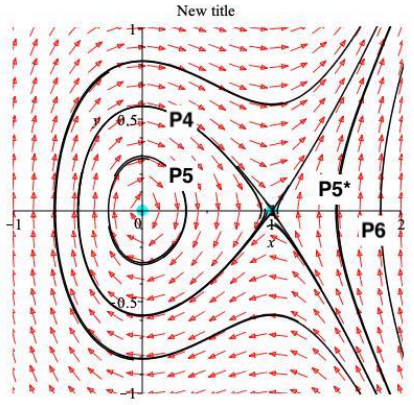

(b)

Figure 4: (a) For even $n$ and $n a_{0}>0,(0,0)$ is center point and $\left(-\left(\frac{1}{a_{1}}\right)^{\frac{1}{n}}, 0\right),\left(\left(\frac{1}{a_{1}}\right)^{\frac{1}{n}}, 0\right)$ are unstable saddle point. (b) For odd $n$ and $n a_{0}>0,(0,0)$ is center point and $\left(\left(\frac{1}{a_{1}}\right)^{\frac{1}{n}}, 0\right)$ are unstable saddle point.

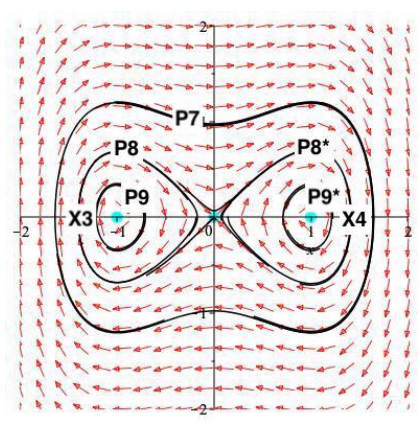

(a)

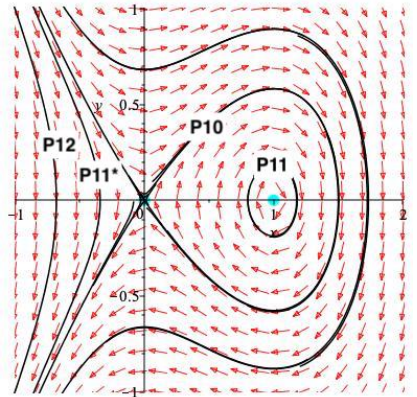

(b)

Figure 5: (a) For even $n$ and $n a_{0}<0,(0,0)$ is saddle point and $\left(-\left(\frac{1}{a_{1}}\right)^{\frac{1}{n}}, 0\right),\left(\left(\frac{1}{a_{1}}\right)^{\frac{1}{n}}, 0\right)$ are center points. (b) For odd $n$ and $n a_{0}<0,(0,0)$ is saddle point and $\left(\left(\frac{1}{a_{1}}\right)^{\frac{1}{n}}, 0\right)$ is center point. 
(II) When $0<h<h^{*}$, system 5.78 has three periodic orbits $P 2, P 2^{*}$ and $P 2^{* *}$.

(III) When $h=h^{*}$, system (5.78) has two heteroclonic orbits P3 and P3*.

(IV) When $h<0$ or $h>h^{*}$, system (5.78) does not have close orbit.

Proposition 5.2. When $n$ is odd number and $n a_{0}>0$, we have

(I) When $h=h^{*}$, system 5.78) has a homoclinic orbit P4.

(II) When $0<h<h^{*}$, system 5.78 has two periodic orbits P5 and $P 5^{*}$.

(III) When $h=0$, system (5.78) has a periodic orbit P6.

(IV) When $h<0$ or $h>h^{*}$, system (5.78) does not have close any orbit.

Now according to Fig. 5, we have following propositions.

Proposition 5.3. When $n$ is even number and $n a_{0}<0$, we have

(I) When $h>0$, system (5.78) has a periodic orbit P7.

(II) When $h=0$, system (5.78) has two homoclinic orbits P8 and P8*.

(III) When $-h^{*}<h<0$, system (5.78) has two periodic orbits P9 and P9*.

(IV) When $h \leq-h^{*}$, system (5.78) does not have close any orbit.

Proposition 5.4. When $n$ is odd number and $n a_{0}<0$, we have

(I) When $h=0$, system (5.78) has a homoclinic orbit P10.

(II) When $-h^{*}<h<0$, system (5.78) has two periodic orbits P11 and P11*.

(III) When $h=-h^{*}$, system 5.78 has a periodic orbit P12.

(IV) When $h<-h^{*}$ or $h>0$, system (5.78) does not have close any orbit.

The qualitative theory of dynamical systems suggest that a smooth homoclinic orbit of a traveling wave equation results in a smooth solitary wave solution of a partial differential equation. A smooth heteroclinic orbit of a traveling wave equation results in a smooth kink wave solution or a unbounded wave solution.. Similarly, a periodic orbit of a traveling wave equation results in a periodic traveling wave solution of a partial differential equation. So we have following propositions.

Proposition 5.5. When $n$ is even number and $n a_{0}>0$, we have

(I) For $h=0,(1.4$ has four periodic blow up wave solutions corresponding to the orbits P1 and $P 1^{*}$ in Fig $4 a$.

(II) For $0<h<h^{*}$, (1.4) four periodic blow up wave solutions and a periodic wave solution corresponding to the orbits orbits $P 2, P 2^{*}$ and $P 2^{* *}$ in Fig $4 a$.

(III) When $h=h^{*}$, 1.4 has two kink solitary wave solutions and two unbounded wave solutions corresponding to orbits $\mathrm{P} 3$ and $\mathrm{P}^{*}$ in Fig 4 a.

Proposition 5.6. When $n$ is odd number and $n a_{0}>0$, we have

(I) When $h=h^{*}$, (1.4) has a solitary wave solution corresponding to the homoclinic orbit P4 in Fig. 4 b.

(II) When $0<h<h^{*}$, (1.4) has a periodic wave solution and two periodic blow up wave solutions corresponding to the two periodic orbits $P 5$ and $P 5^{*}$ in Fig. $4 b$. 
(III) When $h=0$, (1.4) has two periodic blow up wave solutions corresponding to a periodic orbit P6 in Fig. 46.

Now according to Fig. 5, we have following propositions.

Proposition 5.7. When $n$ is even number and $n a_{0}<0$, we have

(I) When $h>0$, (1.4) has two periodic wave solutions corresponding to the periodic orbits P7 in Fig. $5 a$.

(II) When $h=0$, (1.4) has two solitary wave solutions corresponding to the homoclinic orbits P8 and P8* in Fig. $5 a$.

(III) When $-h^{*}<h<0$, 1.4 has periodic wave solutions corresponding to the periodic orbits P9 and P9* in Fig. 5 a.

Proposition 5.8. When $n$ is odd number and $n a_{0}<0$, we have

(I) When $h=0,1.4$ has a solitary wave solution corresponding to the homoclinic orbit P10 in Fig. $5 b$.

(II) When $-h^{*}<h<0,(1.4$ has a periodic wave solution and two periodic blow up wave solutions corresponding to the periodic orbits $P 11$ and $P 11^{*}$ in Fig. $5 b$.

(III) When $h=-h^{*}$, (1.4) has two periodic blow up wave solutions corresponding to the periodic orbit P12 in Fig. $5 b$.

\subsection{Travelling wave solutions}

Firstly,let us consider the case when $n$ is even and $n a_{0}>0$, we obtain the explicit expressions for travelling wave solutions for $(1.4)$. From phase portrait, we have two special orbits $P 1$ and $P 1^{*}$ which have the same value of Hamiltonian as that of center point $(0,0)$. In $(X, Y)$ plane the expressios of the orbits are given by

$$
Y= \pm \sqrt{\frac{2 a_{1}}{a_{0}(n+2)} X^{n+2}-\frac{1}{a_{0}} X^{2}}
$$

Substituting (5.92) into $\frac{d X}{d \sigma}=Y$ and integrating along $P 1$ and $P 2$, we have

$$
\begin{aligned}
& \pm \int_{X_{i}}^{X} \frac{1}{\sqrt{\frac{2 a_{1}}{a_{0}(n+2)} s^{n+2}-\frac{1}{a_{0}} s^{2}}} d s=\int_{0}^{\sigma} d \sigma \\
& \pm \int_{X}^{\infty} \frac{1}{\sqrt{\frac{2 a_{1}}{a_{0}(n+2)} s^{n+2}-\frac{1}{a_{0}} s^{2}}} d s=\int_{0}^{\sigma} d \sigma
\end{aligned}
$$

where $i=1,2$.

Evaluating the integrals, we have

$$
\begin{aligned}
& X= \pm\left(\frac{(n+2)}{2 a_{1}} \sec ^{2}\left(\frac{n}{2 \sqrt{a_{0}}} \sigma\right)\right)^{\frac{1}{n}}, \\
& X= \pm\left(\frac{(n+2)}{2 a_{1}} \csc ^{2}\left(\frac{n}{2 \sqrt{a_{0}}} \sigma\right)\right)^{\frac{1}{n}} .
\end{aligned}
$$

Using the above expressions with $V=X$ and Eqs. 3.24, 4.37, the solutions of main equation (1.4) are given by

$$
\begin{aligned}
& v=\frac{1}{b}\left[1 \mp\left(\frac{n+2}{2 a_{1}} \sec ^{2}\left(\frac{n}{2 \sqrt{a_{0}}}(x-\mu t)\right)\right)^{\frac{1}{n}}\right], \\
& v=\frac{1}{b}\left[1 \mp\left(\frac{n+2}{2 a_{1}} \csc ^{2}\left(\frac{n}{2 \sqrt{a_{0}}}(x-\mu t)\right)\right)^{\frac{1}{n}}\right],
\end{aligned}
$$


where $a_{0}=\frac{\delta^{2}}{12}$ and $a_{1}=\frac{\mu^{2} C_{0} L}{n+1}$.

Now, let us consider the case when $n$ is even and $n a_{0}<0$, we obtain the explicit expressions for travelling wave solutions for 1.4). Again from phase portrait, we have two homoclinic orbits $P 8$ and $P 8^{*}$ intersecting at the saddle point $(0,0)$. In $(X, Y)$ plane the expressios of the orbits are given by

$$
Y= \pm \sqrt{\frac{2 a_{1}}{a_{0}(n+2)} X^{n+2}-\frac{1}{a_{0}} X^{2}} .
$$

Substituting 5.92 into $\frac{d X}{d \sigma}=Y$ and integrating along orbits $P 8$ and $P 8^{*}$, we have

$$
\pm \int_{X_{i}}^{X} \frac{1}{\sqrt{\frac{2 a_{1}}{a_{0}(n+2)} s^{n+2}-\frac{1}{a_{0}} s^{2}}} d s=\int_{0}^{\sigma} d \sigma,
$$

where $i=3,4$.

Evaluating the integrals, we have

$$
X= \pm\left(\frac{n+2}{a_{1}\left(1-\cosh \left(\frac{n}{\sqrt{-a_{0}}} \sigma\right)\right)}\right)^{\frac{1}{n}},
$$

Using the above expressions with $V=X$ and Eqs. (3.24), (4.37), the singular solitary wave solutions of main equation (1.4) are given by

$$
v= \pm\left(\frac{n+2}{a_{1}\left(1-\cosh \left(\frac{n}{\sqrt{-a_{0}}}(x-\mu t)\right)\right)}\right)^{\frac{1}{n}}
$$

where $a_{0}=\frac{\delta^{2}}{12}$ and $a_{1}=\frac{\mu^{2} C_{0} L}{n+1}$.

\section{Conclusion}

In this work, Nonlinear transmission line model (NLTL) with arbitrary power law nonlinearity and the constant capacitor voltage dependence has been studied through application of Lie symmetry analysis. For both the cases i.e., $C(v)=C_{0}(1-b v)$ and $C(v)=C_{0}$ of NLTL, infinitesimal symmetries are obtained and partial differential equations are reduced to ordinary differential equations (ODEs). Furthermore, variety of new exact solutions in the form of trigonometric functions, hyperbolic functions solutions are also obtained.

Additionally, corresponding to one reduction of NLTL with power law nonlinearity, phase plane analysis is carried out. This results in various propositions where the various solution existance structure are examined that depends upon the parameter values. Finally some more solutions of NLTL model in the form of periodic singular waves and singular soliton waves are obtained. Graphical representation of some of solutions is also given and observed effect of parameters in graphs is discussed.

\section{Compliance with ethical standards}

Conflict of interest: The authors declare that they have no conflict of interest.

\section{References}

[1] Ehsan Afshari, Harish S Bhat, Ali Hajimiri, and Jerrold E Marsden. Extremely wideband signal shaping using one-and two-dimensional nonuniform nonlinear transmission lines. Journal of Applied Physics, 99(5):054901, 2006. 
[2] AH Arnous, M Mirzazadeh, and M Eslami. Exact solutions of the Drinfel'd-Sokolov-Wilson equation using Bäcklund transformation of Riccati equation and trial function approach. Pramana, 86(6):1153-1160, 2016.

[3] Gerd Baumann. Non-classical Symmetries of Partial Differential Equations. In Symmetry Analysis of Differential Equations with Mathematica®, pages 365-391. Springer, 2000.

[4] A Biswas, EV Krishnan, P Suarez, AH Kara, and Sachin Kumar. Solitary waves and conservation laws of Bona-Chen equations. Indian Journal of Physics, 87(2):169-175, 2013.

[5] George Bluman and Stephen Anco. Symmetry and integration methods for differential equations, volume 154. Springer Science \& Business Media, 2008.

[6] Si-Jia Chen, Wen-Xiu Ma, and Xing Lü. Bäcklund transformation, exact solutions and interaction behaviour of the $(3+1)$-dimensional Hirota-Satsuma-Ito-like equation. Communications in Nonlinear Science and Numerical Simulation, 83:105135, 2020.

[7] Roman Cherniha, Mykola Serov, and Oleksii Pliukhin. Nonlinear reaction-diffusion-convection equations: Lie and conditional symmetry, exact solutions and their applications. CRC Press, 2017.

[8] Peter A Clarkson and Martin D Kruskal. New similarity reductions of the Boussinesq equation. Journal of Mathematical Physics, 30(10):2201-2213, 1989.

[9] Robert Conte, Micheline Musette, and A Michel Grundland. Bäcklund transformation of partial differential equations from the Painlevé-Gambier classification. ii. Tzitzeica equation. Journal of Mathematical Physics, 40(4):2092-2106, 1999.

[10] MT Darvishi, M Najafi, and AM Wazwaz. New extended rational trigonometric methods and applications. Waves in Random and Complex Media, 30(1):5-26, 2020.

[11] G Ebadi, A Mojaver, S Johnson, S Kumar, and A Biswas. Dynamics of dispersive topological solitons and its perturbations. Indian Journal of Physics, 86(12):1115-1129, 2012.

[12] MM El-Borai, HM El-Owaidy, Hamdy M Ahmed, and AH Arnous. Exact and soliton solutions to nonlinear transmission line model. Nonlinear Dynamics, 87(2):767-773, 2017.

[13] MM El-Borai, AA Zaghrout, and AM Elshaer. Exact solutions for nonlinear partial differential equations by using the extended multiple Riccati. vol, 9:370-381, 2011.

[14] Shimin Guo and Yubin Zhou. The extended G'/G-expansion method and its applications to the Whitham-Broer-Kaup-like equations and coupled Hirota-Satsuma KdV equations. Applied Mathematics and Computation, 215(9):3214-3221, 2010.

[15] Ryogo Hirota. The direct method in soliton theory, volume 155. Cambridge University Press, 2004.

[16] Nail H Ibragimov and Robert Gilmore. Handbook of Lie group analysis of differential equations; applications in engineering \& physical sciences. Mathematical Intelligencer, 19(1):68, 1997.

[17] Anwar Ja'afar Mohamad Jawad, Marko D Petković, and Anjan Biswas. Modified simple equation method for nonlinear evolution equations. Applied Mathematics and Computation, $217(2): 869-877,2010$.

[18] E Kengne, Boris A Malomed, ST Chui, and WM Liu. Solitary signals in electrical nonlinear transmission line. Journal of mathematical physics, 48(1):013508, 2007.

[19] EV Krishnan, Sachin Kumar, and Anjan Biswas. Solitons and other nonlinear waves of the Boussinesq equation. Nonlinear Dynamics, 70(2):1213-1221, 2012. 
[20] Sachin Kumar. Painlevé analysis and invariant solutions of Vakhnenko-Parkes (VP) equation with power law nonlinearity. Nonlinear Dynamics, 85(2):1275-1279, 2016.

[21] SI Mostafa. Analytical study for the ability of nonlinear transmission lines to generate solitons. Chaos, Solitons \& Fractals, 39(5):2125-2132, 2009.

[22] Peter J Olver. Applications of Lie groups to differential equations, volume 107. Springer Science \& Business Media, 2000.

[23] Lev Vasil'evich Ovsiannikov. Group analysis of differential equations. Academic press, 2014.

[24] Jiri Patera and Pavel Winternitz. Subalgebras of real three-and four-dimensional Lie algebras. Journal of Mathematical Physics, 18(7):1449-1455, 1977.

[25] Ali Sardar, SM Husnine, Syed Tahir Raza Rizvi, M Younis, and K Ali. Multiple travelling wave solutions for electrical transmission line model. Nonlinear Dynamics, 82(3):1317-1324, 2015.

[26] Karanjeet Singh, Rajesh Kumar Gupta, and Sachin Kumar. Benjamin-Bona-Mahony (BBM) equation with variable coefficients: similarity reductions and Painlevé analysis. Applied Mathematics and Computation, 217(16):7021-7027, 2011.

[27] Dig Vijay Tanwar and Abdul-Majid Wazwaz. Lie symmetries, optimal system and dynamics of exact solutions of (2+ 1)-dimensional kp-bbm equation. Physica Scripta, 95(6):065220, 2020.

[28] Abdul-Majid Wazwaz. Optical bright and dark soliton solutions for coupled nonlinear schrödinger (cnls) equations by the variational iteration method. Optik, 207:164457, 2020.

[29] Abdul-Majid Wazwaz and Lakhveer Kaur. Complex simplified hirota's forms and lie symmetry analysis for multiple real and complex soliton solutions of the modified kdv-sine-gordon equation. Nonlinear Dynamics, 95(3):2209-2215, 2019.

[30] Yu-Hang Yin, Wen-Xiu Ma, Jian-Guo Liu, and Xing Lü. Diversity of exact solutions to a (3+ 1)-dimensional nonlinear evolution equation and its reduction. Computers $\mathscr{E}$ Mathematics with Applications, 76(6):1275-1283, 2018.

[31] Muhammad Younis and Safdar Ali. Solitary wave and shock wave solitons to the transmission line model for nano-ionic currents along microtubules. Applied Mathematics and Computation, 246:460-463, 2014.

[32] Muhammad Younis, Syed Tahir Raza Rizvi, and Safdar Ali. Analytical and soliton solutions: nonlinear model of nanobioelectronics transmission lines. Applied Mathematics and Computation, 265:994-1002, 2015.

[33] Sheng Zhang, Jing-Lin Tong, and Wei Wang. A generalized (G'/G)-expansion method for the mKdV equation with variable coefficients. Physics Letters A, 372(13):2254-2257, 2008. 
Figures

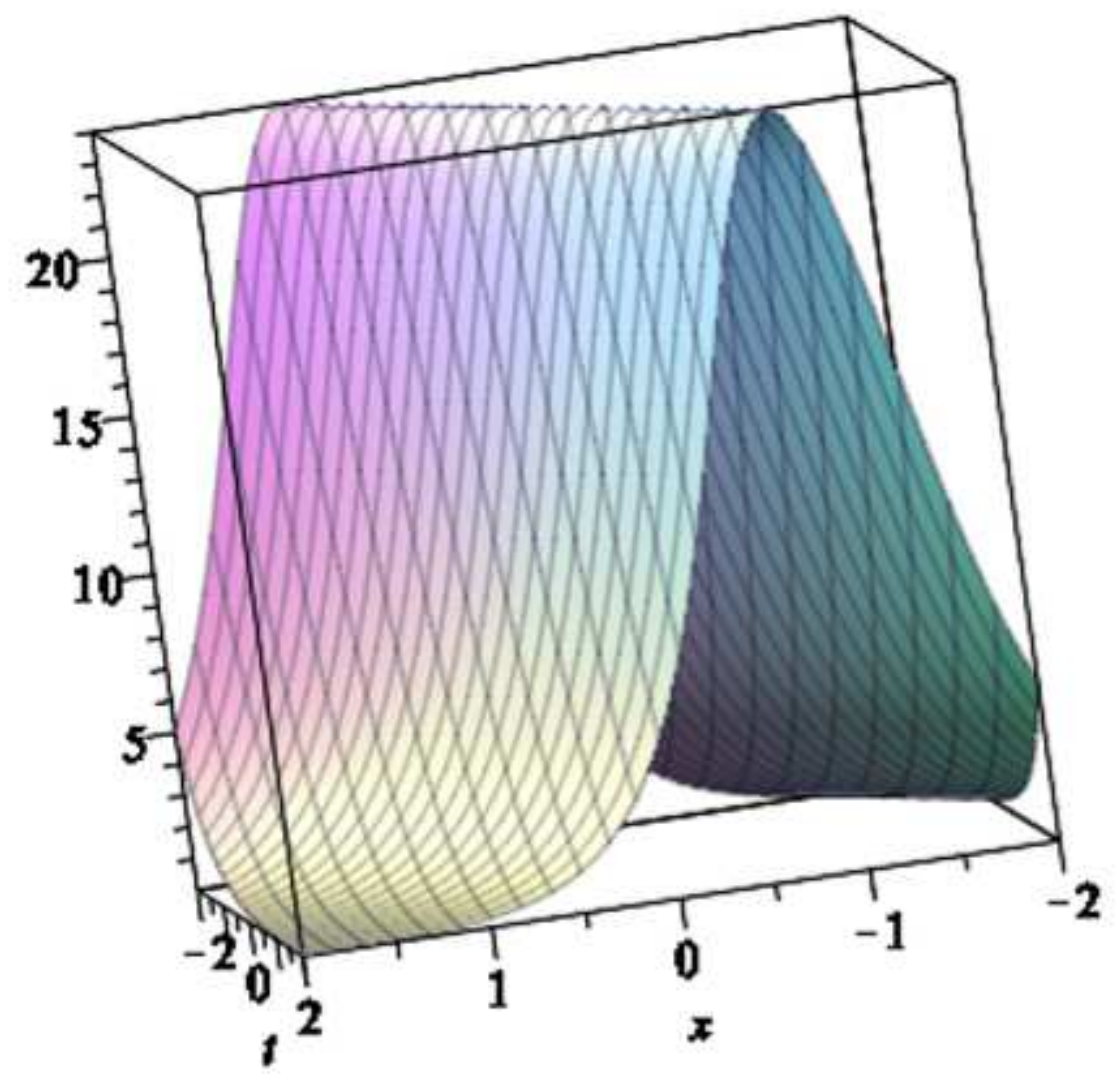

Figure 1

Soliton solution (4.59)

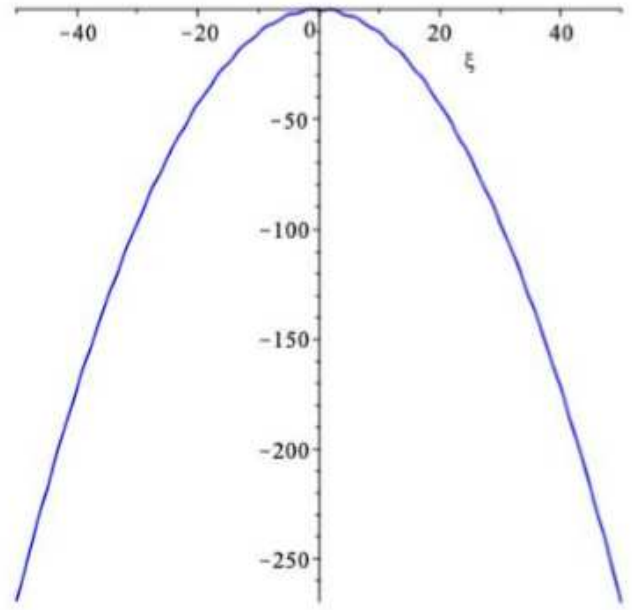

(a) As $\xi \rightarrow \pm \infty$

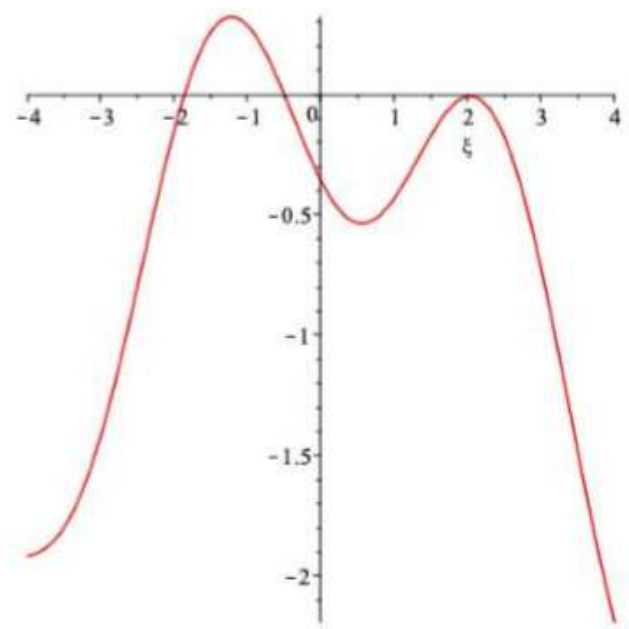

(b) For transient value of $\xi$ 
Figure 2

For $\mathrm{A} 1=\mathrm{A} 2=\mathrm{C} 9=\mathrm{C} 3=\delta=\mathrm{C} 0=1, \mathrm{~L}=1.2, \mu=0.8$, graphs of the solutions (4.74).

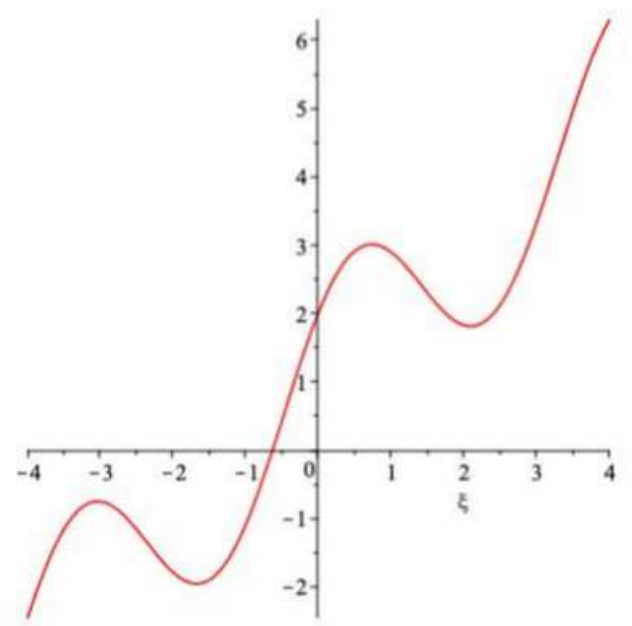

(a) Quasi periodic solution

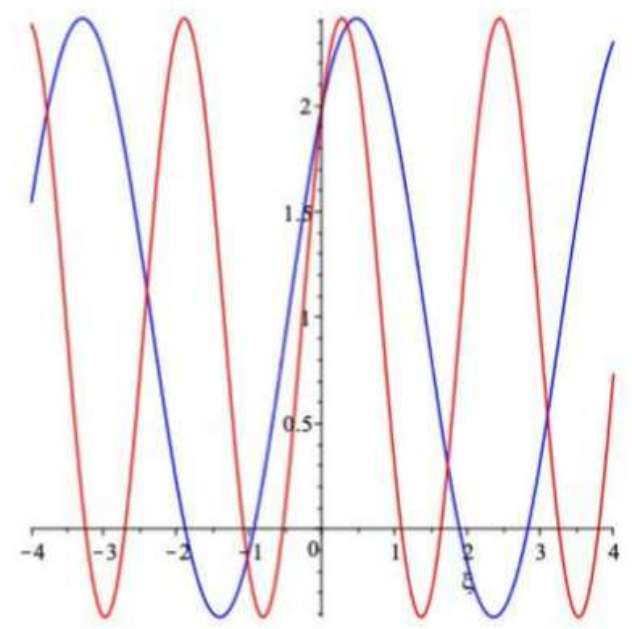

(b) Periodic solution

Figure 3

Graphs of the solution (4.76) (a) For $\mathrm{A} 1=\mathrm{A} 2=\mathrm{A} 3=\mathrm{A} 4=\delta=\mathrm{C} 0=1, \mathrm{~L}=1.2, \mu=0.8$ (b) For $\mathrm{A} 1=\mathrm{A} 3=\mathrm{A} 4=$ $\delta=C 0=1, A 2=0, L=1.2$ and $\mu=0.5, \mu=0.8$ is shown corresponding to red, blue colors, respectively.

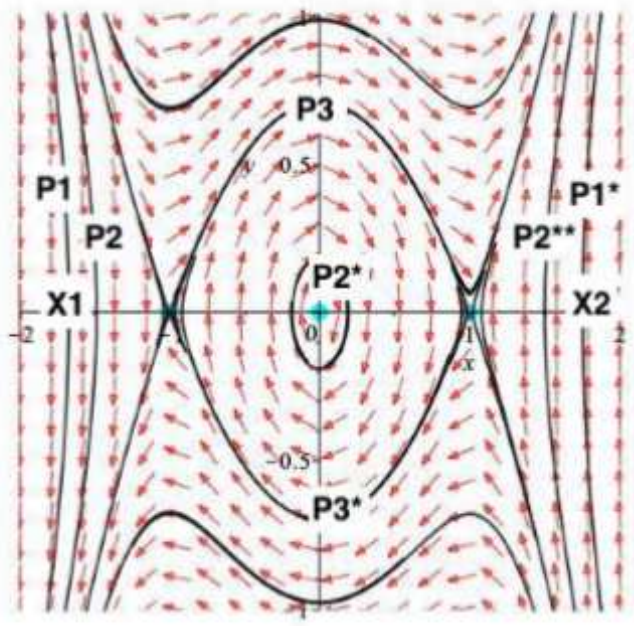

(a)

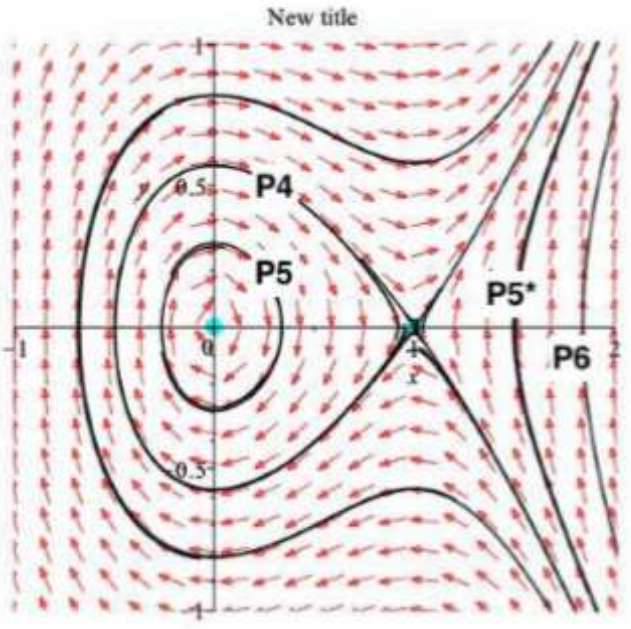

(b)

\section{Figure 4}

Please see the Manuscript PDF file for the complete figure caption 


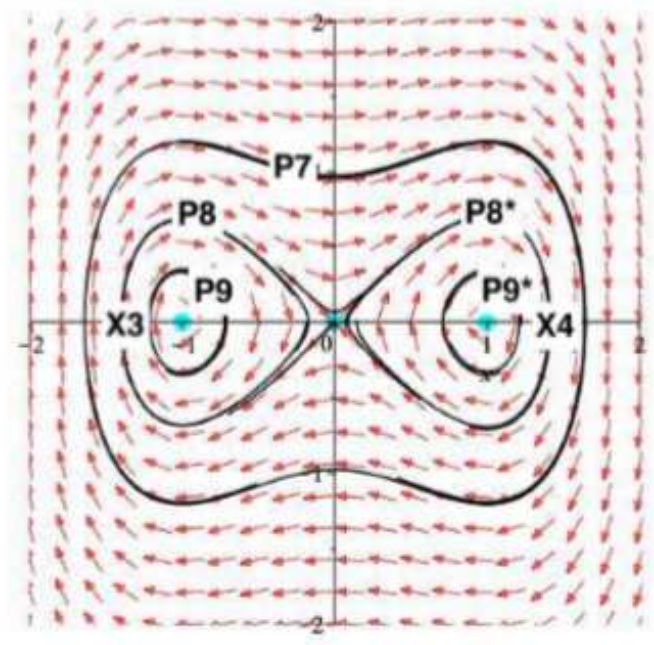

(a)

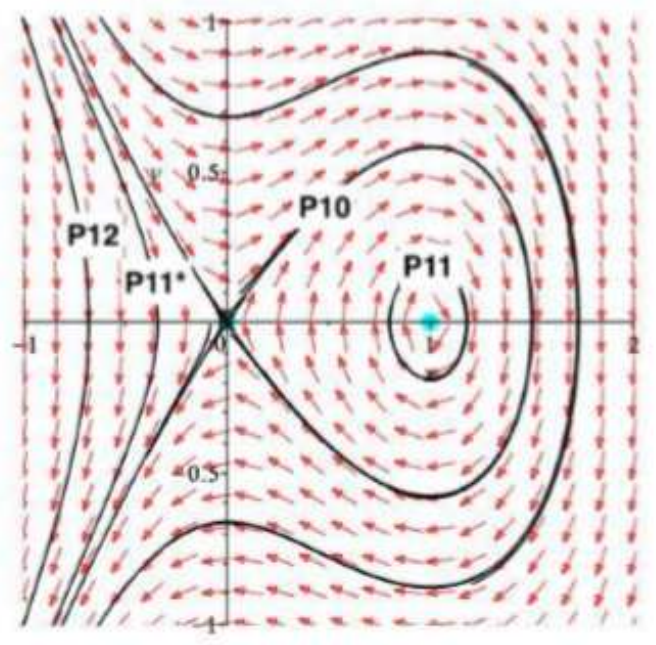

(b)

Figure 5

Please see the Manuscript PDF file for the complete figure caption 